\title{
Class of self-dual models in three dimensions
}

\author{
A. de Souza Dutra* \\ UNESP/Campus de Guaratinguetá-DFQ, Av. Dr. Ariberto Pereira da Cunha, 333 Guaratinguetá, São Paulo, Brazil \\ C. P. Natividade \\ UNESP/Campus de Guaratinguetá-DFQ, Av. Dr. Ariberto Pereira da Cunha, 333 Guaratinguetá, São Paulo, Brazil \\ and Instituto de Física-Universidade Federal Fluminense, Av. Litorânea S/N, Boa Viagem, Niterói, \\ Rio de Janeiro, Rio de Janeiro, Brazil \\ (Received 26 June 1998; published 21 December 1999)
}

\begin{abstract}
In the present paper we introduce a hierarchical class of self-dual models in three dimensions, inspired in the original self-dual theory of Towsend-Pilch-Nieuwenhuizen. The basic strategy is to explore the powerful property of the duality transformations in order to generate a new field. The generalized propagator can be written in terms of the primitive one (first order), and also the respective order and disorder correlation functions. Some conclusions about the "charge screening" and magnetic flux were established.
\end{abstract}

PACS number(s): 11.10.Ef, 11.10.Kk, 11.15.-q

From the mathematical point of view, topological theories in three dimensions contain a rich variety of models which have received much attention in the last years. One of them is the self-dual model [1], which presents a close connection with the well established Chern-Simons (CS) theory [2]. This fact could be confirmed in different ways: for instance, by comparing the Green functions of the Maxwell-ChernSimons (MCS) theory and the self-dual (SD) model [2,3], by inspecting the constraint structure of each model [3], or through the bosonization of the massive Thirring model in three dimensions, which is related to the MCS theory in the large mass limit [4]. In this last case, the equivalence between both models has been obtained starting from a careful analysis of the partition function and was improved later through the calculation of higher order derivative terms [5].

In the present work, we shall introduce a hierarchical family of dual models in three dimensions, related to the original SD model. The mathematical structure of the SD theory offers an alternative way of building up $N$ families of dual models. In the final step, a master Lagrangian density corresponding to a higher order derivative model is generated. A very interesting aspect of this model is the existence of an isomorphism between its observables and those obtained in its first order form. This fact can be proved through different procedures: first, in the canonical analysis of the fields and their momenta, by using the treatment of order reduction [6]. In what follows, we will use a method developed in a series of papers [7] (see also [8] for related works), in order to describe the magnetic flux and charge on the plane $\left(x^{1}, x^{2}\right)$ through two dual operators $(\mu, \sigma)$, called disorder and order operators, respectively.

In order to implement our alternative model, let us begin exploring the mathematical structure of the self-dual fields. In this sense, let us consider the duality transformation of the primary field $A_{\mu}^{(N)}$,

\footnotetext{
*Email address: dutra@ feg.unesp.br
}

$$
A_{\mu}^{(N)}=\epsilon_{\mu \alpha \beta} \partial^{\alpha} A_{\beta}^{(N+1)},
$$

where the index $N$ is an integer which identifies the family of the respective self-dual field. The relation (1) gives rise to the possibility of generating a class of Lagrangian densities indexed by $N$.

Let us start our study by considering the following Lagrangian density:

$$
L_{1}=-\frac{a}{4}\left(F_{\mu \nu}\right)^{2}+b \partial_{\mu} F^{\mu \lambda} \partial^{\nu} F_{\nu \lambda}+\theta \epsilon^{\mu \nu \rho} \partial_{\sigma} A_{\mu} \partial_{\nu} \partial^{\sigma} A_{\rho},
$$

which has been examined recently [9], with $a, b$, and $\theta$ defined in it. Now, we are going to show that the Lagrangian density appearing in Eq. (2) is a higher order extension from the Proca-Chern-Simons one:

$$
L_{1}^{(0)}=-\frac{a}{2} A_{\mu}^{(0)} A^{\mu(0)}+\frac{b}{2}\left(F_{\mu \nu}^{(0)}\right)^{2}+\theta \epsilon^{\mu \nu \rho} A_{\mu}^{(0)} \partial_{\nu} A_{\rho}^{(0)} .
$$

By using the transformation (1), with $N=0$, it is lengthy but straightforward to show that we arrive at the Lagrangian density (2). The propagators can be related among them, since $\left\langle A_{\mu}^{(0)} A_{\nu}^{(0)}\right\rangle=-\epsilon_{\mu \alpha \beta} \epsilon_{\nu \grave{\alpha} \dot{\beta}}\left\langle\partial^{\alpha} A_{\beta}^{(1)} \partial^{\grave{\alpha}} A_{\dot{\beta}}^{(1)}\right\rangle$.

From the above considerations it becomes clear that the results obtained here can be generalized from the $N$ order to the $(N+1)$ one. Therefore, from the basic Lagrangian density given by Eq. (3), we can build up the following generic higher-order Lagrangian density:

$$
\begin{aligned}
L^{(N)}= & \frac{(-1)^{(N-1)}}{4} F_{\mu \nu}^{(N)} \square^{(N-1)}(a \square+b) F^{\mu \nu(N)} \\
& -(-1)^{(N-1)} \theta \epsilon^{\mu \nu \rho} A_{\mu}^{(N)} \partial_{\nu} \square^{(N)} A_{\rho}^{(N)} .
\end{aligned}
$$

The above Lagrangian density belongs to a class such that the first one is related to the bosonization of the massive 
Thirring model [5]. In order to simplify the calculation of the canonical momenta, we are going to define the quantities

$$
f_{\mu} \equiv \sqrt{\square^{N-1}} A_{\mu}, \dot{f}_{\mu} \equiv \sqrt{\square^{N-1}} \dot{A}_{\mu},
$$

where $\square^{-n}=\int\left[d^{3} k /(2 \pi)^{3}\right]\left(k^{2}\right)^{-n} e^{i k x}$. Now, if we take the rescaling $\square^{-n} \rightarrow(\square-\Omega)^{-n}$, and take $\Omega \rightarrow 0$ at the end of the calculations, the expansion in powers of the d'Alambertian can be employed by acting on the fields. Consequently, we can derive the canonical momenta associated to independent variables $\left(f_{\mu}, \dot{f}_{\mu}\right)$ in a natural way,

$$
\delta S^{(n)}=\int d^{3} x \sum_{n=0}^{\infty} \frac{d}{d t}\left(\pi_{\nu}^{(n)} \delta f^{\nu}+S_{\nu}^{(n)} \delta \dot{f}^{\nu}\right),
$$

where now the action $S^{(n)}=\int d t L^{(n)}$ is the reduced form from those in Eq. (4). Therefore, the momenta become

$$
\begin{aligned}
\pi^{\nu(N)}= & (-1)^{N-1}\left\{b f^{0 \nu(N)}+a\left(\partial^{k} \partial^{\lambda} f_{\lambda}^{0(N)} \delta_{k}^{\nu}-\partial^{0} \partial^{\lambda} f_{\lambda}^{\nu(N)}\right)\right. \\
& \left.-2 \theta \epsilon^{\mu \lambda \nu} \partial_{\lambda} \partial^{\nu} f_{\mu}\right\}, \\
s^{\nu(N)}=( & -1)^{N-1} 2 a\left(\partial_{\mu} f^{\mu \nu(N)}-\delta_{0}^{\nu} \partial_{\rho} f^{0 \rho(N)}\right)-\theta \epsilon^{0 \lambda \nu} \partial_{0} f_{\lambda}^{(N)},
\end{aligned}
$$

which relates physical quantities from $N$ theory with the first-order one. From the above equations we conclude that the basic commutators of the present theory in the Coulomb gauge are

$$
\begin{aligned}
{\left[f_{l}^{(N)}(\bar{x}), \pi_{k}^{(N)}(\bar{y})\right]_{x^{0}=y^{0}}=} & \sqrt{\nabla^{2(N-1)}}\left[A_{l}^{(N)}(\bar{x}), \pi_{k}^{(N)}(\bar{y})\right]_{x^{0}=y^{0}} \\
= & -i(-1)^{N-1}\left\{b \delta_{l k} \delta^{2}(\bar{x}-\bar{y})\right. \\
& \left.+\left(b+a \nabla^{2}\right) \partial_{l} \partial_{k} G(\bar{x}-\bar{y})\right\} \\
{\left[\dot{f}_{l}(\bar{x}), s_{k}^{(N)}(\bar{y})\right]_{x^{0}=y^{0}}=} & -i(-1)^{N-1} b \delta_{l k} \delta^{2}(\bar{x}-\bar{y}) \\
= & \sqrt{\nabla^{2(N-1)}}\left[\dot{A}_{l}^{(N)}(\bar{x}), s_{k}^{(N)}(\bar{y})\right]_{x^{0}=y^{0}},
\end{aligned}
$$

and also

$$
\begin{aligned}
{\left[\pi_{l}^{(N)}(\bar{x}), \pi_{k}^{(N)}(\bar{y})\right]_{x^{0}=y^{0}}=} & -i \theta(-1)^{N-1}\left(b+a \nabla^{2}\right) \\
& \times \epsilon_{i k} \partial_{l} \partial^{i} \delta^{2}(\bar{x}-\bar{y}),
\end{aligned}
$$

where $G(\bar{x}, \bar{y})$ obeys the equation

$$
\left(b+a \nabla^{2}\right) \nabla^{2} G(\bar{x}, \bar{y})=\delta^{2}(\bar{x}-\bar{y}) .
$$

Here we remark that the application of the expansion of $\sqrt{\square^{N-1}}$ on the above brackets, extracts the temporal part of the d'Alambertian operator.

The Lagrangian density (4) permits us to infer the corresponding form of the photon propagator in momentum space

$$
\begin{aligned}
D_{\mu \nu}^{(N)}(k)= & \frac{-1}{k^{2 N}\left(f^{2}-4 \theta^{2} k^{2}\right)}\left\{\left(a+\frac{b}{k^{2}}\right) P_{\mu \nu}+2 i \epsilon^{\mu \lambda \nu} k_{\lambda}\right\} \\
& -\frac{\xi}{f} \frac{k_{\mu} k_{\nu}}{k^{2 N+2}}
\end{aligned}
$$

where the last term corresponds to a gauge fixing. By using Fourier transform we can obtain the equivalent propagator in the coordinate space. Here, we adopt $P_{\mu \nu}=k^{2} g_{\mu \nu}-k_{\mu} k_{\nu}$ and $f \equiv b-a k^{2}$. Hence, if we fix some parameters in the original Lagrangian density given by Eq. (4) like $a \equiv 4 \alpha^{2}, b=1$ and $N=1$, we obtain the photon correlation function

$$
\begin{aligned}
\left\langle A_{\mu}(x) A_{\nu}(y)\right\rangle^{\text {dual }}= & {\left[\left(1-4 \alpha^{2} \square\right) P_{\mu \nu}+i \bar{\theta} \epsilon_{\mu \nu \alpha} \partial^{\alpha}\right] } \\
& \times\left(\frac{1}{\left[\frac{\bar{\theta}}{\alpha}\left(1-\frac{\alpha^{2}}{\bar{\theta}^{2}}\right)\right]^{2}}-1\right)\left(\frac{4 \alpha^{2}}{\bar{\theta}^{2}}\right) \\
& \times \frac{e^{-(\bar{\theta} / \alpha)\left(1-\alpha^{2} / \bar{\theta}^{2}\right) R}}{4 \pi R}-\frac{1}{4 \pi} \\
& \times \xi \partial_{\mu} \partial_{\nu}\left(2 \alpha-\frac{R}{4}\right)
\end{aligned}
$$

with $\bar{\theta} \equiv i \theta, 4 \alpha^{2}<\bar{\theta}^{2}$ and "dual" stands for the generalized model defined through the propagator of the Lagrangian density. The above equation represents the photon correlation function of the problem mentioned in Ref. [9].

At this point, we are able to extract a very interesting and useful result about the order-disorder correlation functions, starting from Eq. (12). We remember to the reader that the order-disorder formalism has been introduced first by Kadanoff and Ceva [10] in order to discuss the existence of a generalized statistics. Posteriorly this was extended to the continuum quantum field theory [11]. This procedure has been applied to some models in $(2+1)$ dimensions by using a new interpretation of the operators that generate the statistics. Now, over the plane $\left(x^{1}, x^{2}\right)$, the Maxwell theory has a nontrivial value for the topological charge associated with the identically conserved current $J^{\mu}=\epsilon^{\mu \nu \rho} \partial_{\nu} A_{\rho}$. The magnetic flux content correspondent to $J^{\mu}$ is described by a nonlocal operator (vortex operator) $\mu(x)$ defined on a certain curve $C$. The correlation function $\langle\mu(1) \mu(2)\rangle$ of the disorder operator is given as Euclidean functional integrals. In the same way, we can define the charge bearing operator $\sigma(x)$ as being a dual version of $\mu$.

In order to give a better understanding of the role of order-disorder correlation functions, we will take as an example the case of the Maxwell-Chern-Simons theory, since its photon propagator in the coordinate space will be useful in the following.

The order correlation function for the MCS theory is defined in terms of the following Euclidean functional integral: 


$$
\begin{aligned}
\left\langle\sigma(x) \sigma^{*}(y)\right\rangle= & Z^{-1} \int D A_{\mu} \exp \left\{-\int d^{3} z\right. \\
& \left.\times\left[\frac{1}{2} A_{\mu}\left(P^{\mu \nu}+\theta C^{\mu \nu}+G^{\mu \nu}\right) A_{\nu}+C_{\mu} A^{\mu}\right]\right\}
\end{aligned}
$$

where $P^{\mu \nu} \equiv-\square \delta^{\mu \nu}+\partial^{\mu} \partial^{\nu}, C^{\mu \nu} \equiv-i \epsilon^{\mu \alpha \nu} \partial_{\alpha}$ and $G^{\mu \nu}$ is the usual gauge fixing term. Here we adopt an external field $C_{\mu}$.

Integrating over $A_{\mu}$ we readily obtain

$$
\begin{aligned}
\left\langle\sigma(x) \sigma^{*}(y)\right\rangle= & \exp \left\{\frac{1}{2} \int d^{3} z d^{3} z^{\prime} C_{\mu}(z)\right. \\
& \left.\times\left[P^{\mu \nu}+\theta C^{\mu \nu}+G^{\mu \nu}\right]^{-1} C_{\nu}\left(z^{\prime}\right)\right\}
\end{aligned}
$$

with $\left[P^{\mu \nu}+\theta C^{\mu \nu}+G^{\mu \nu}\right]^{-1}=\left\langle A_{\mu}(x) A_{\nu}(y)\right\rangle_{M C S}$ being the Euclidean propagator of the $A_{\mu}$ field in MCS theory. Its explicit expression in the coordinate space is given by

$$
\begin{aligned}
\left\langle A_{\mu}(x) A_{\nu}(y)\right\rangle_{M C S}= & {\left[P^{\mu \nu}+i \theta \epsilon^{\mu \alpha \nu} \partial_{\alpha}\right]\left[\frac{1-e^{-\theta R}}{4 \pi \theta^{2} R}\right] } \\
& -\lim _{m \rightarrow 0} \xi \partial^{\mu} \partial^{\nu}\left[\frac{1}{m}-\frac{R}{8 \pi}\right] .
\end{aligned}
$$

Before going on, we should remark that $\left\langle\sigma(x) \sigma^{*}(y)\right\rangle$ is not a gauge invariant quantity. The reason is that under a formal gauge transformation $A_{\mu} \rightarrow A_{\mu}+\Lambda$, the charge operator changes to $\sigma^{\prime}=\exp (2 \pi i \Lambda(x)) \sigma$. In this way, going back to Eq. (14), we must extract the gauge independent part of $\left\langle\sigma(x) \sigma^{*}(y)\right\rangle$. This will be achieved by inserting the gauge independent part of $\left\langle A_{\mu}(x) A_{\nu}^{*}(y)\right\rangle_{M C S}$, namely $\delta^{\mu \nu}$ and $\epsilon^{\mu \alpha \nu}$ proportional terms. At the end of the calculations, it can be shown that only the diagonal part of $\left\langle A_{\mu}(x) A_{\nu}^{*}(y)\right\rangle_{M C S}$ proportional to $\delta^{\mu \nu} \square$ contributes to the order correlation function. Therefore we obtain the following expression:

$$
\begin{aligned}
& \langle\sigma(x) \sigma *(y)\rangle_{M C S} \\
& =\exp \left\{\left\langle A_{\mu}(x) A_{\nu}^{*}(y)\right\rangle_{\substack{\text { diag } \\
=0}}\right\} \exp \left(\frac{e^{-\theta R}}{4 \pi R}\right) \\
& =\exp \left\{\frac{\pi a^{2}}{\bar{\theta}}\left[e^{-\bar{\theta} R}-1\right]\right\} \Rightarrow\left\langle\sigma_{R}(x) \sigma_{R} *(y)\right\rangle \\
& =\exp \left\{\frac{\pi a^{2}}{\bar{\theta}} e^{-\bar{\theta} R}\right\}
\end{aligned}
$$

where the renormalization $\sigma_{R} \equiv \sigma e^{\pi a^{2} / 2 \bar{\theta}}$ was adopted and $a$ is a charge parameter. As a consequence, $\lim _{R \rightarrow \infty}\left\langle\sigma_{R}(x) \sigma_{R} *(y)\right\rangle=1$, which reflects the screening of the charge associated with the mass generation for the gauge field induced by the CS term.
Now, going back to our model, we begin considering the limit which excludes the Podolsky term, $\bar{\theta}^{2} \gg \alpha^{2}$, in Eq. (12),

$$
\begin{aligned}
\left\langle A_{\mu}(x) A_{\nu}(y)\right\rangle_{\xi=0}^{d u a l} \cong & {\left[P_{\mu \nu}+2 i \bar{\theta} \epsilon_{\mu \nu \alpha} \partial^{\alpha}\right]\left(\frac{1}{\bar{\theta}^{2}}-1\right) } \\
& \times\left(\frac{4}{\bar{\theta}^{2}}\right) \frac{e^{-\bar{\theta} R}}{4 \pi R},
\end{aligned}
$$

in order to compare it with results of the MCS case. By examining the diagonal part of the above propagator we have

$$
\begin{aligned}
\left\langle A_{\mu}(x) A_{\nu}(y)\right\rangle^{d u a l} & \cong \square \delta_{\mu \nu}\left(\frac{1}{\bar{\theta}^{2}}-1\right)\left(\frac{4}{\bar{\theta}^{2}}\right) \frac{e^{-\bar{\theta} R}}{4 \pi R} \\
& \cong\left(\frac{1}{\bar{\theta}^{2}}-1\right) \frac{e^{-\bar{\theta} R}}{4 \pi R}+\text { extra terms } \\
& =-\left(\frac{1}{\bar{\theta}^{2}}-1\right)\left\langle A_{\mu}(x) A_{\nu}(y)\right\rangle_{M C S}
\end{aligned}
$$

where "extra terms" are proportional to the $\delta$-functions. From Eqs. (15) and (17) we expect that

$$
\begin{aligned}
\left\langle\sigma_{R}(x) \sigma_{R} *(y)\right\rangle^{d u a l} \cong & \exp \left[\left(\frac{1}{\bar{\theta}^{2}}-1\right)^{-1}\right] \\
& \times\left\langle\sigma_{R}(x) \sigma_{R} *(y)\right\rangle_{M C S},
\end{aligned}
$$

for $R \rightarrow \infty$

$$
\left\langle\sigma_{R}(x) \sigma_{R} *(y)\right\rangle^{d u a l} \rightarrow \text { const. }
$$

Therefore the order correlation function, which is associated with charge screening, in our model has a similar behavior to that of the MCS theory. The result given by Eq. (18) expresses the charge screening, which in this case is

$$
Q^{d u a l}=\int d^{2} z J^{0}=\theta \int d^{2} z \nabla_{\xi}^{2} \epsilon_{i j} \partial_{z}^{i} A^{i}(z, \xi),
$$

which differs from the usual MCS charge by a second order derivative operator. Here $J_{\mu} \equiv \partial^{\nu} J_{\mu \nu}$ defined in Eq. (23) below. Note that the presence of the differential operators in $Q^{\text {dual }}$ does not alter the long range distance behavior of the order correlation function when compared with MCS theory.

Now, in order to build the disorder correlation function $\langle\mu(1) \mu(2)\rangle$ in our model, we begin defining the vortex operator which is associated to the magnetic flux on the plane $\left(x^{1}, x^{2}\right)$. This is obtained by coupling a certain external field $W_{\mu}$ to the dual current through

$$
J_{\theta}^{\mu \nu} \equiv F^{\mu \nu}-\frac{\theta \square \epsilon^{\mu \nu \alpha}}{\left(1-4 \alpha^{2} \square\right)} A_{\alpha},
$$

which comes from the equation of motion. The generalized disorder operator can be written as

$$
\mu_{\theta}^{d u a l}(x)=\exp \left\{-i b \int d^{3} z J_{\theta}^{\mu \nu} W_{\mu \nu}\right\},
$$


where $W^{\mu \nu}$ is an external tensor field, $W_{\mu \nu} \equiv \partial_{\mu} W_{\nu}$ $-\partial_{\nu} W_{\mu}$, which would be coupled to the conserved current $J_{\theta}^{\mu \nu}$ in order to obtain the correct correlation function

$$
\begin{aligned}
\left\langle\mu_{\theta}(x) \mu *_{\theta}(y)\right\rangle^{d u a l}= & \int D A_{\mu} \exp \left\{-\int d^{3} z\left[\frac{1}{2} A^{\mu} D_{\mu \nu}^{d u a l} A^{\nu}\right.\right. \\
& \left.\left.+A^{\mu} D_{\mu \nu(G C S)} W^{\nu}+\frac{1}{4}\left(W_{\mu \nu}\right)^{2}\right]\right\},
\end{aligned}
$$

with $G C S$ standing for generalized Chern-Simons, and $D_{\mu \nu}^{d u a l}$ is given by

$$
\begin{gathered}
D_{\mu \nu}^{d u a l} \equiv\left(1-4 \alpha^{2} \square\right) P_{\mu \nu}-\xi \partial_{\mu} \partial_{\nu}-\theta \epsilon_{\mu \alpha \nu} \partial^{\alpha} \square, \\
D_{\mu \nu}^{G C S} \equiv P_{\mu \nu}-\theta \epsilon_{\alpha \nu} \partial^{\alpha} \square\left(1-4 \alpha^{2} \square\right)^{-1},
\end{gathered}
$$

where $P_{\mu \nu} \equiv-\square \delta_{\mu \nu}+\partial_{\mu} \partial_{\nu}$. Now, if we consider the action of the operators $P_{\mu \nu}$ and $\theta \epsilon_{\mu \alpha \nu} \partial^{\alpha} \square$ over $W_{\mu \nu}$, this gives rise to

$$
\begin{gathered}
P_{\mu \nu} W^{\mu} \rightarrow Z_{\nu}=\int d \lambda_{\nu} \delta^{3}(z-\lambda) \\
\frac{\theta \square \epsilon_{\mu \alpha \nu} \partial^{\alpha} W^{\mu}}{\left(1-4 \alpha^{2} \square\right)} \rightarrow U_{\nu}=\theta \int d \lambda^{\mu} \epsilon_{\mu \alpha \nu} \partial^{\alpha} \square\left(1-4 \alpha^{2} \square\right)^{-1} \\
\times \delta^{3}(z-\lambda) .
\end{gathered}
$$

Therefore, after integration over the field $A_{\mu}$ in Eq. (22) we get

$$
\begin{aligned}
\left\langle\mu_{\theta}(x) \mu *_{\theta}(y)\right\rangle_{N=1}^{\text {dual }}= & \exp \left\{\frac { 1 } { 2 } \int d ^ { 3 } z d ^ { 3 } \dot { z } \left(Z_{\mu}(z, x, y)\right.\right. \\
& \left.+U_{\mu}(z, x, y)\right)\left\langle A_{\mu}(x) A *_{\nu}(y)\right\rangle^{d u a l} \\
& \times\left(Z_{\nu}(\dot{z}, x, y)+U_{\nu}(\dot{z}, x, y)\right) \\
& \left.-\frac{1}{4}\left(W_{\mu \nu}\right)^{2}\right\}
\end{aligned}
$$

where $\left\langle A_{\mu}(x) A *{ }_{\nu}(y)\right\rangle^{\text {dual }}$ is given by Eq. (12). Now, if we now turn our attention to the fact that in the limit $\theta \gg 4 \alpha^{2}$, the photon correlation function is given by Eq. (18) and the field $U_{\nu}$ will not depend on the factor $4 \alpha^{2}$, we will have

$$
\begin{aligned}
\left\langle\mu_{\theta}(x) \mu *_{\theta}(y)\right\rangle_{N=1}^{d u a l}= & \exp \left\{\frac{1}{2}\left(1-\frac{1}{\bar{\theta}^{2}}\right) \int d^{3} z d^{3} z\left(Z_{\mu}+\widetilde{U}_{\mu}\right)\right. \\
& \times\left\langle A_{\mu} A *_{\nu}\right\rangle^{M C S}\left(Z_{\nu}+\widetilde{U}_{\nu}\right) \\
& \left.-\frac{1}{4}\left(W_{\mu \nu}\right)^{2}\right\}
\end{aligned}
$$

with $\widetilde{U}_{\nu}=\theta \int_{x, L} d \lambda^{\mu} \epsilon_{\mu \alpha \nu} \partial^{\alpha} \square \delta^{3}(z-\lambda)$.

Now, we note that up to $\square$ term into $\widetilde{U}_{\nu}$ field, the integrand of the above equation corresponds to the correlation function of MCS theory. However, since $\left\langle A_{\mu}(x) A *_{\nu}(y)\right\rangle_{M C S}$ depends on $1 /|z-z|$ the contractions which involve the field $\widetilde{U}_{\nu}$ give rise to delta functions $\delta^{3}(z-\lambda)$ and $\delta^{3}(z-\eta)$ such that the line integral over $d \lambda_{\mu}$ and $d \eta_{\mu}$ vanishes. This means that the integrand of the Eq. (30) corresponds to that of the MCS theory or,

$$
\left\langle\mu_{\theta}(x) \mu *_{\theta}(y)\right\rangle^{d u a l}=\exp ^{\left(1-1 / \theta^{2}\right)}\left\langle\mu_{\theta}(x) \mu *_{\theta}(y)\right\rangle^{M C S} .
$$

Therefore, since the behavior of the vortex correlation function operator in the MCS theory for very large distances $|x-y| \rightarrow \infty$ is a constant, indicating that $\mu_{\theta}$ does not create genuine vortex excitations, we expect the same behavior for the dual theory.

For a future program, we intend to investigate the possible connection with the interesting formalism developed by Barci et al., where a mapping was made among some models in three dimensions [12]. This was done by using a nonlinear redefinition of the gauge field, in contrast to the linear selfdual transformation used in this work.

The authors are grateful to CNPq and FAPESP for partial financial support, and to D. Dalmazi for discussions.
[1] P.K. Towsend, K. Pilch, and P. van Nieuwenhuizen, Phys. Lett. 136B, 32 (1984); 137B, 443(E) (1984).

[2] S. Deser, R. Jackiw, and S. Templeton, Phys. Rev. Lett. 48, 975 (1982); Ann. Phys. (N.Y.) 140, 372 (1982); S. Deser and R. Jackiw, Phys. Lett. 139B, 371 (1984).

[3] R. Banerjee, H.J. Rothe, and K.D. Rothe, Phys. Rev. D 52, 3750 (1995); Nucl. Phys. B447, 3750 (1995).

[4] E. Fradkin and F.A. Schaposnik, Phys. Lett. B 338, 253 (1994).

[5] A. de Souza Dutra and C.P. Natividade, Mod. Phys. Lett. A 14, 307 (1999).

[6] R.P.L.G. Amaral and E.C. Marino, J. Phys. A 25, 5183 (1992).

[7] E.C. Marino, Phys. Rev. D 38, 3194 (1988); Ann. Phys. (N.Y.) 224, 225 (1993); Int. J. Mod. Phys. A 10, 4311 (1995); Mod. Phys. Lett. A 11, 1985 (1996); Phys. Rev. D 55, 5234 (1997).
[8] J. Frölich and A. Marchetti, Lett. Math. Phys. 16, 347 (1988); G.W. Semenoff and P. Sodano, Nucl. Phys. B328, 753 (1989); M. Lüscher, ibid. B326, 557 (1989).

[9] A. de Souza Dutra and C.P. Natividade, Mod. Phys. Lett. A 11, 775 (1996); see also S. Deser and R. Jackiw, Phys. Lett. B 451, 73 (1999).

[10] L.P. Kadanoff and H. Ceva, Phys. Rev. B 3, 3518 (1971).

[11] E.C. Marino and J.A. Swieca, Nucl. Phys. B: Field Theory Stat. Syst. 170 [FS1], 175 (1980); E.C. Marino, B. Schroer, and J.A. Swieca, ibid. 200 [FS4], 473 (1982).

[12] V.E.R. Lemes, C. Linhares de Jesus, G.A.G. Sasaki, S.P. Sorella, L.C.Q. Vilar, and O.S. Ventura, Phys. Lett. B 418, 324 (1998); D.G. Barci, V.E.R. Lemes, C. Linhares de Jesus, M.B.D. Silva Maia Porto, S.P. Sorella, and L.C.Q. Vilar, Nucl. Phys. B524, 765 (1998). 